\title{
Homology modeling and substrate binding studies of human P-glycoprotein
}

\author{
J. Pimthon ${ }^{1,2^{*}}$, R. Dechaanontasup ${ }^{1,2}$, C. Ratanapiphop ${ }^{1,2}$, C. Phromprasert ${ }^{1,2}$ \\ ${ }^{1}$ Department of Pharmaceutical Chemistry, Faculty of Pharmacy, Mahidol University, \\ 447 Sri-Ayudhya Road, Bangkok 10400, Thailand \\ ${ }^{2}$ Center of Excellence for Innovation in Drug Design and Discovery, Faculty of Pharmacy, \\ Mahidol University, 447 Sri-Ayudhya Road, Bangkok 10400, Thailand
}

\begin{abstract}
Multidrug-resistance (MDR) in cancer cells often relates to the overexpression of P-glycoprotein (P-gp), resulting in increased efflux of chemotherapy from cancer cells. A clear understanding of P-gp substrate binding will lead to the development of selective P-gp inhibitors, which resensitize cancer cells to standard chemotherapy. Unfortunately, the three-dimensional structure of human P-glycoprotein has not yet been available. In this investigation, homology model of human P-gp was developed based on a recent refined structure of mouse P-gp (PDB: 4M1M). The models were further assessed by Ramachandran plot in PROCHECK, ProSA-web Z-score and QMEAN score. The results indicated that the proposed models were reliable for further binding site and docking studies. Using AutoDock-based blind docking protocol, the probable binding sites for the known substrates rhodamine B, daunorubicin, colchicine, and Hoechst 33342 were identified and in very good agreement with the available side-directed mutagenesis studies. The binding location of the cytotoxic drug vinblastine was identified and characterized. The docking result indicated that vinblastin and verapamil shared overlapping sites on P-gp, composed of residue Leu65, Met69, Ile340, Phe983, Tyr953, and Met986. This might aid in understanding how verapamil, an inhibitor of P-gp, effectively enhanced cytotoxicity of vinblastine against P-gp-mediated MDR. Our observations suggested that human P-gp model derived from 4M1M could better explain the binding of human P-gp substrates/ inhibitors. This model served as a starting point to gain knowledge of P-gp drug-binding region(s) and to identify novel P-gp inhibitors that might have a potential to overcome MDR in cancer therapy.
\end{abstract}

Keyword: P-glycoprotein, Homology model, Molecular docking, Drug binging sites, Interaction.

\section{INTRODUCTION}

P-glycoprotein (P-gp), also called multidrug resistance protein (MDR1), is one of the well-characterized efflux pumps among the highly conserved ATP-binding cassette (ABC) superfamily of proteins. It plays a pivotal role as a barrier for xenobiotic as well as eliminates xenobiotic from systemic circulation. P-gp is of considerable clinical importance as often involves in the multi-drug resistance phenotype overexpressed by several cancer cell lines ${ }^{1}$.

It is well known that P-gp substrates vary greatly in size, structure and function, which suggest multiple binding sites within P-gp ${ }^{1}$.
Therefore, defining the residues involved in the interaction between P-gp and its substrate may shed some light on how P-gp can recognize and transport a broad range of structurally diverse compounds. Additionally, such knowledge is important for developing the potent, selective and specific P-gp inhibitors. However, to the best of our knowledge, there is no crystal structure of either human P-gp or the complex of P-gp with naturally occurring substrates available. Recently, there has been a progress in using computational approaches to enhance our understanding of multi-drug efflux mechanisms ${ }^{2}$. To rationally design drugs, it typically requires structural templates of the drug targets. In the 
lack of an experimentally established crystal structure of a given protein, good homology models are valuable drug discovery tools ${ }^{3}$. Although the crystallographic structure of human P-gp is not yet available, there are a number of published P-gp crystal structures from other different species. Among the popular experimentally determined structures for developing a P-gp homology model, Caenorhabditis elegans (C. elegans) P-gp (PDB entry: 4F4C) shares less sequence identity to human P-gp $(46 \%)$ than that of the original mouse P-gp structure (PDB ID: 3G5U) (87\%). However, homology models, based on both templates, yield a reliable structure for substrate binding studies and enhance its functional understanding of human P-gp ${ }^{4}$. Recently, Li et al. ${ }^{5}$ reported a refined structure of mouse P-gp with a significant improvement in the protein geometry. Their findings suggested the use of murine P-gp (PDB code: $4 \mathrm{M} 1 \mathrm{M}$ ) as a more appropriate template to generate the homology model of human $\mathrm{P}$-gp in order to minimize errors arising from evolutionary divergence.

Current studies aimed at developing a better human P-gp homology model by using the refined crystal structure of mouse as a template. To evaluate the quality of the model, the known P-gp substrates were docked into the predicted protein model and compared the binding modes with related experimental studies. Additionally, the interactions between the homology model of P-gp and a selected anticancer drug were also monitored. The obtained results are considered to be useful in the study the nature of the drugbinding site(s) of P-gp. The model can also be utilized to identify novel P-gp substrates or inhibitors. Furthermore, the optimized threedimensional (3D) structure of human P-gp can be exploited to predict pharmacokinetic, efficacy, and safety profiles of drugs, which are both necessary and advantageous in drug discovery and development process.

\section{MATERIALS AND METHODS}

\subsection{Homology modeling of human P-gp}

Homology modeling of the inward-facing state of human P-gp was executed as previously reported $^{6}$. In brief, the sequence of human P-gp was obtained from the universal protein resource (entry: P08183) ${ }^{7}$. The template for sequence alignment was identified through searching human P-gp on PDB using the BLASTP program provided by UniProt with default parameters ${ }^{8}$. Accordingly, the crystal structure of murine P-gp (PDB ID: 4M1M, $\mathrm{X}$-RAY diffraction resolution: $3.8 \AA$ ) was taken as the templates to model human P-gp. Sequence alignment of the PDB structures versus human P-gp sequence was performed on $\mathrm{ClustalW}^{8}$, using a gap penalty of 5 and a gap extension penalty of 0.1 . Subsequently, homology modeling of human P-gp was performed on MODELLER $9 \mathrm{v} 13$ software ${ }^{9}$. The linker region was not modeled. The top 10 best models were selected on the basis of the DOPE score amongst 1000 models generated and evaluated by PROCHECK ${ }^{10}$, ProSA web Z-score ${ }^{11}$ and QMEAN scores ${ }^{12}$. The best template for docking was chosen on the basis of various factors such as Z-score, number of residues in core that fall in generously allowed and disallowed regions in Ramachandran plot. To improve the quality of the model, the secondary structure predicted by PORTER servers ${ }^{13}$ was included. The iterative energy minimizations were performed to refine the obtained homology model.

\subsection{Molecular Docking}

Prior to molecular docking, the hydrogen atoms were added to the generated human P-gp structure by using the REDUCE software ${ }^{14}$. The software was also helped in flipping protein side-chains where necessary. The 3D structures of colchicine, Hoechst 33342, vinblastine, rhodamine $\mathrm{B}$, and daunorubicin were retrieved from RCSB PDB (PDB ID: 4O2B, 130D, 4EB6, 4GLJ, and 110D, respectively). The 2D structure of verapamil was retrieved from the NCBI PubChem database (CID: 2520). These compounds were then converted to their corresponding mol2 (3D format) using open babel software ${ }^{15}$. AutoDockTools v1.5.7 $\mathrm{rc}^{16}$ was used to set the rotatable bonds and add polar hydrogen. Gasteiger charges ${ }^{17}$ were computed and the protein was embedded in a grid box with 
dimensions $106 \times 110 \times 126$, on the mass center of the protein with a grid spacing of $0.375 \AA$. Docking was performed by AutoDock 4.2 program, using the implemented empirical free energy function and the Lamarckian Genetic Algorithm ${ }^{16}$. Because the locations of the ligand in the complex, including to verify the quality of the predicted model, were unclear, AutoDock-based blind docking approach ${ }^{18}$ was utilized to explore the entire surface of protein for possible ligand binding sites. The preferential docking locations of the molecules were defined as the drug-binding pockets. The maximum number of energy evaluations and of generations was set to 10 million and 100 runs, each with a population of 150 individuals, were performed for each calculation. All other parameters were set by default. AutoDockTools was utilized to evaluate the binding conformations to select the conformation of the best fit. The protein-ligand complexes were visualized and analyzed using Discovery Studio Visualizer (BIOVIA, San Diego, CA). It enables identification of non-covalent interactions, such as hydrogen bond, van der Waals, and $\pi-\pi$, formed between the ligand and the receptor. All structural images were generated with Pymol v.1.3 ${ }^{19}$.

\section{RESULTS AND DISCUSSION}

\subsection{Homology model of human P-gp and its validation}

Homology modeling is usually the method of choice when a clear relationship of homology between the sequence of the target protein and at least one known X-ray crystallographic structure is found. In present study, a recent refinement of the crystal structure of murine P-gp (PDB code: 4M1M) was selected as an appropriate template for modeling 3D structure of human P-gp. The quality of its crystal structure was significantly improved, as compared to the original mouse P-gp structure (PDB ID: 3G5U). Moreover, the template had sufficient crystal structure resolution (X-RAY diffraction resolution: $3.8 \AA$ ). The sequence alignment of the Mus musculus (PDB code: $4 \mathrm{M} 1 \mathrm{M}$ ) with the human P-gp portrayed that the template and target shared significant similarity to each other (87\% identical) (Figure 1). The proposed model of human P-gp (Figure 2A) was subjected to structure verification and evaluation using PROCHECK, ProSA web Zscore and QMEAN. From the Ramachandran plot analysis computed with the PROCHECK program, 93.6\%, 4.4\%, 1.4\%, and $0.6 \%$ were found situated in the most favoured region, additional allowed region, generously allowed region, and disallowed region, respectively (Figure 2B). A good quality Ramachandran plot would be expected to have over $90 \%$ in the favoured regions ${ }^{10}$. The total quality G-factor 0.1 indicated a good quality model as the acceptable values of the G-factor in PROCHECK are between 0 and -0.5 , with the best models displaying values close to zero ${ }^{12}$. Furthermore, ProSA-web was used to determine the Z-score values of the predicted homology models. The value was displayed in a plot containing the $Z$ scores of all experimental determined protein structures. It could be used to indicate whether the input structures were within the range of scores that were typically found for native proteins of similar size. The ProSA Z-score of -14.15 (Figure 2C) represented the good quality of the generated model ${ }^{11}$. Moreover, the local model quality of human P-gp was determined in terms of energy function of amino acid residues (Figure 2D). In general, negative values correspond to stable parts of the input structure. The plot revealed a negative value of energy particularly in the windows size of 40 (i.e., the average energy value for 40 amino acid residues at position $i$ ), which confirmed that the predicted model was reliable. The QMEAN score of the model was 0.63 and the Z-score was -1.63 , very close to the value of 0 , determining the good quality of the model ${ }^{12,20}$. Focused on molecular structure of generated human P-gp model, it was observed that the distance between the conserved Cys431 and Cys 1074 residues in the nucleotide-binding domains (NBDs) was 25.3 $\AA$. The value was consistent with the recently disulfide cross-linking study, indicating that the minimal distance between NBDs of apo conformation of human P-gp appeared to be closer (20-25 $\AA$ ) than that of mouse P-gp (36 $\AA)$ and C. elegans P-gp $(54 \AA)^{21}$. In addition, 
Lee et $a l .{ }^{22}$ reported the containing residues, Arg905 and Ser909, of human P-gp were in close proximity to Leu443 and Ser474 by using cysteine disulphide cross-linking experiments. These data correlated well with our predicted homology model (data not shown). Accordingly, our results, in terms of geometry and energy contours, suggested that the generated model of human P-gp was reasonable and reliable for further molecular docking studies.

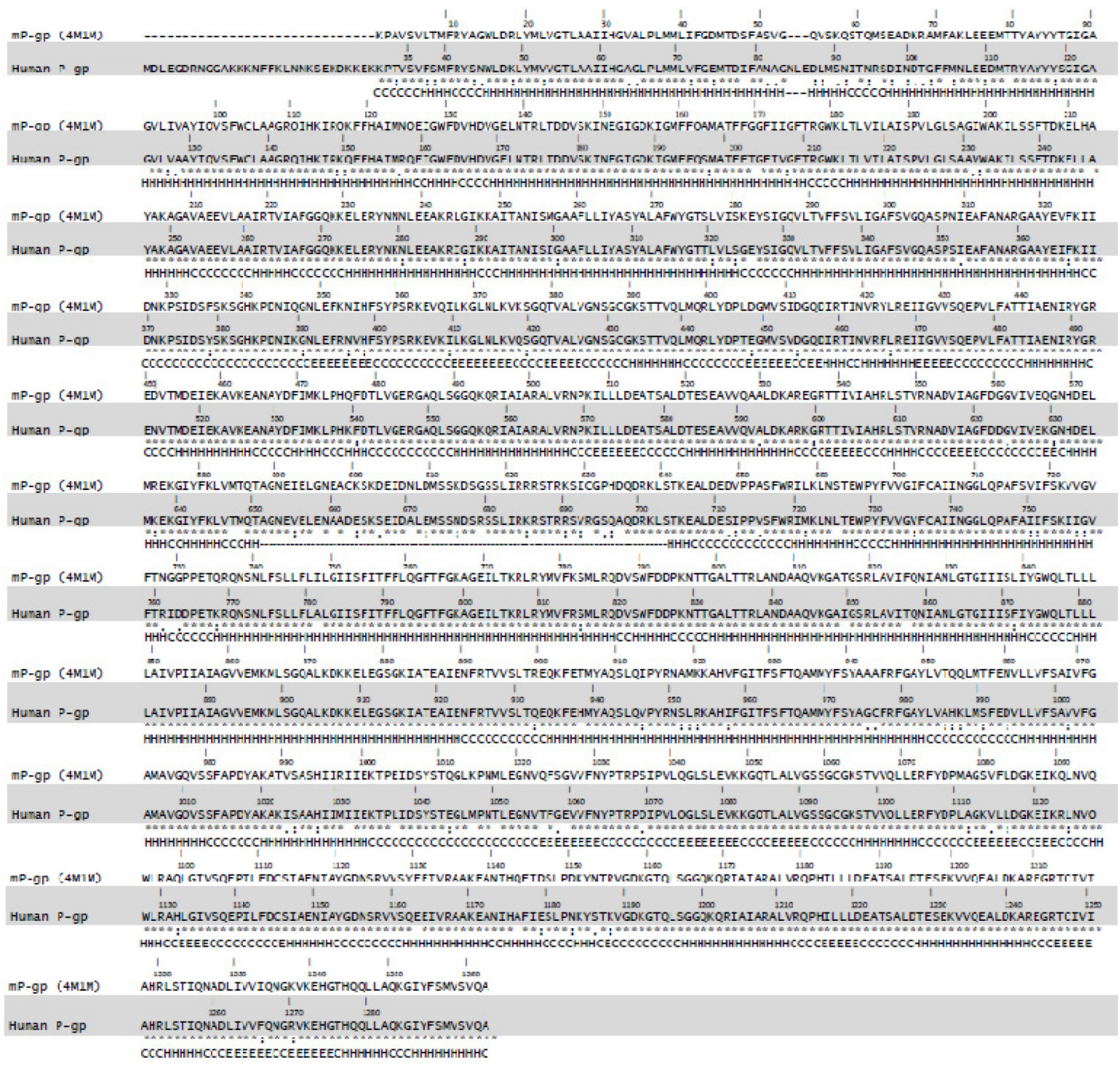

Figure 1. Sequence alignment of human P-gp (target) with murine P-gp (PDB code: 4M1M) (template). Asterisks indicate identical amino acids, dots indicate similar amino acids and colons indicate very similar amino acids. 
(A)

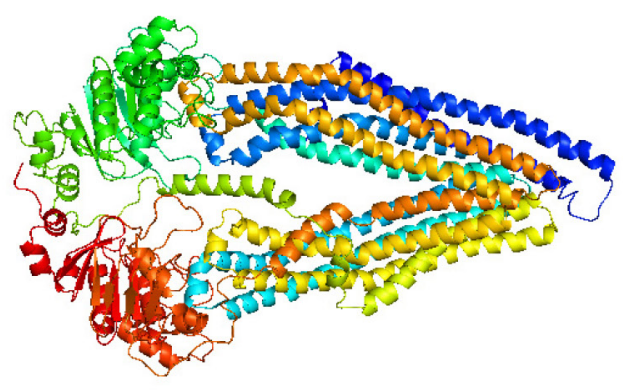

(C)

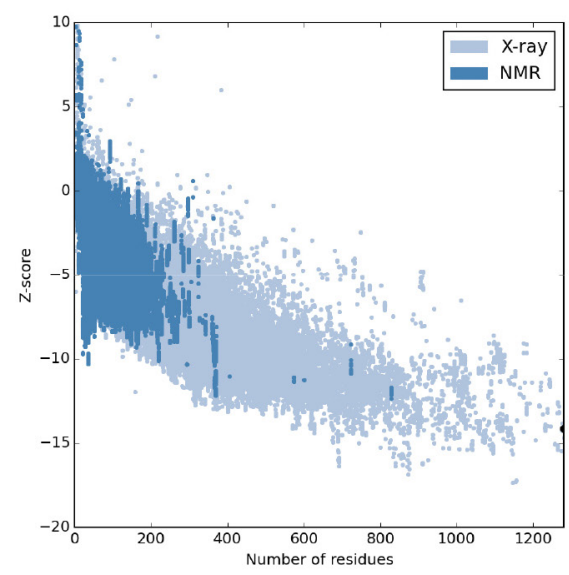

(B)

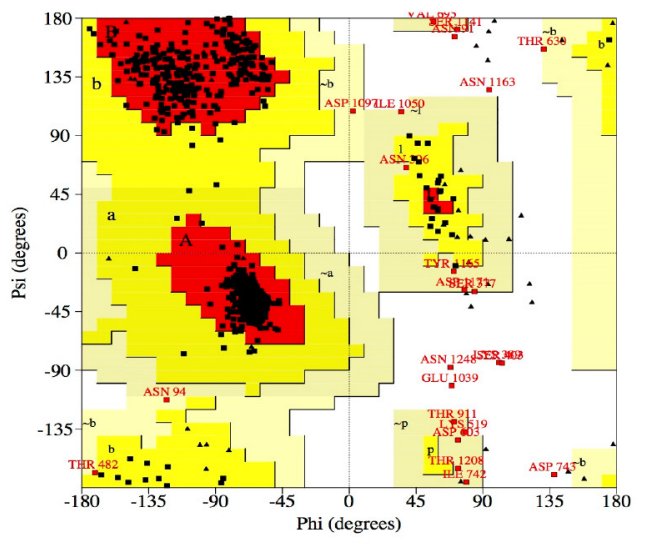

(D)

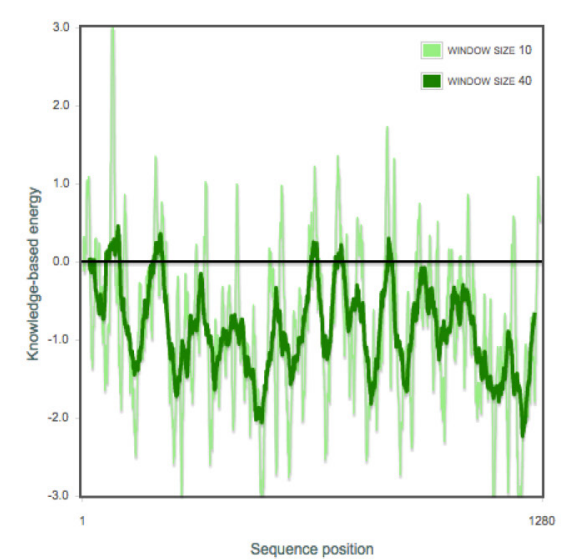

Figure 2. (A) 3D structure of generated human P-gp model. (B) Ramachandran plot (the red, dark yellow, light yellow, and white regions represent the most favoured, additional allowed, generously allowed, and disallowed regions, respectively). (C) ProSA-web Z-scores of all protein chains in PDB determined by X-ray crystallography (light blue) and nuclear magnetic resonance spectroscopy (dark blue) with respect to their length. The Z-score of human P-gp homology model was present in the range represented in black dot. (D) ProSA energy plot calculated for the predicted human P-gp. (For interpretation of the references to color in this figure legend, the reader is referred to web version of this article).

\subsection{Docking characterization of substrates binding sites in human P-gp}

To further assess the accuracy of homology modeling, a number of P-gp substrates were docked against the predicted model and the results were compared with available experimental studies. It was proposed that P-gp contained at least two substrate binding sites, denoted the R-site and the $\mathrm{H}$-site, and that a particular drug might interact with one or both of these sites $^{23}$. In this investigation, the ligand for R-site, rhodamine B and daunorubicin, and the ligand for H-site, Hoechst 33342 and colchicine, were subjected for docking studies. The chemical structures of P-gp ligands are shown in Figure 3. 
<smiles>CCN(CC)c1ccc2c(-c3ccccc3C(=O)O)c3ccc(=[N+](CC)CC)cc-3oc2c1</smiles><smiles>COc1cccc2c1C(=O)C1C(O)=C3C(=C(O)C1C2=O)C[C@](O)(C(C)=O)C[C@@H]3O[C@H]1C[C@H](N)[C@H](O)[C@H](C)O1</smiles><smiles></smiles>

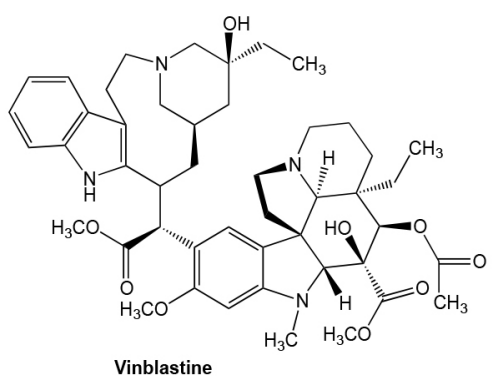<smiles>CCCCCCOc1ccc(-c2nc3ccc(-c4nc5ccc(N6CC[NH+](C)CC6)cc5[nH]4)cc3[nH]2)cc1</smiles><smiles>COc1ccc(CCN(C)CCC[C@](C#N)(c2ccc(OC)c(OC)c2)C(C)C)cc1OC</smiles>

Figure 3. The chemical structures of the P-gp ligands that were used in this study

According to the blind docking results, the binding mode of rhodamine $\mathrm{B}$, a highly hydrophobic compound, was strongly influenced by van der Waals interactions, e.g., with Tyr310, Phe343, Phe978, and Met986. The binding sites of rhodamine $\mathrm{B}$ and its contacted residues are shown in Figure 4A. The best pose showed binding energy of about $-6.9 \mathrm{kcal} / \mathrm{mol}$. The observation showed a qualitative agreement with cysteine-scanning mutagenesis in combination with thiol-labeling approach, suggested that the cystein mutations of Ile340, Ala841, Leu 1975, Va1981, and Va1982 were significantly protected from inhibition by methanethiosulfonate (MTS)-rhodamine by pretreatment with rhodamine $\mathrm{B}^{24}$. Therefore, these residues were possibly involved in the rhodamine drug-binding site. In addition, the docking results revealed that a benzene moiety of rhodamine B made van der Waals contacts with Tyr 310 and Phe343. This finding was in excellent agreement with the results of site-directed mutagenesis studies, indicating that the phenylanine-343-arginine mutation reduced the apparent affinity of P-gp for rhodamine, in turn, underlined the significant contribution of the residue to the rhodamine drug-binding pocket ${ }^{25}$.

For the complex of daunorubicin with human P-gp model (Figure 4B), docking results revealed that daunorubicin located at the hydrophobic pocket, contributed by Leu65, Met69, Phe72, Ile340, Phe343, Phe336, Phe983, 
and Met986, overlapped to rhodamine binding site. A hydrogen bond formation between Tyr310 and amino sugar moiety (daunosamine) was observed. Aromatic $\pi-\pi$ interactions were also detected between daunorubicinone moiety and Phe72, Phe336. Our finding agreed well with the previous studies stating that daunorubicin bound at the R-site similar to rhodamine $\mathrm{B}^{23}$. Daunorubicin also showed similar binding energy $(-6.54 \mathrm{kcal} / \mathrm{mol})$ to that observed for rhodamine B $(-6.9 \mathrm{kcal} / \mathrm{mol})$. This could explain the experimental observation on a direct competition between rhodamine and daunorubicin at the R-site, exhibited a decrease in the efflux rate of rhodamine in correlation with daunorubicin concentration $^{23}$.

Furthermore, molecular docking of Hoechst 33342 into the human P-gp homology model was also performed. Hoechst 33342 was known substrate commonly interacting with $\mathrm{H}$-site of human P-gp ${ }^{26}$. Fluorescence spectroscopy studies suggested that Hoechst 33342 binding site was located at a very hydrophobic transmembrane region. However, there is no experimental information regarding the binding residues of Hoechst 33342. Docking results showed that the closest residue to the substrate Hoechst 33342 binding site was Phe72, Gln195, Thr199, Phe336, Ile340, Gly341, Ala342, Phe343, Ser344, Val345, Gly346, Gln347, Ser349, Pro350, Tyr950, Tyr953, Phe957, Leu975, Phe978, Ser979, and Phe983. The piperazine ring moiety of Hoechst 33342 was surrounded by polar amino acid residues, Gly346 and Gln347. The benzimidazole moiety was situated in a nonpolar environment, contributed by Ile340 and Phe343. The NH of benzimidazole moiety showed hydrogen bonding with Tyr953. The ethoxyphenyl moiety was surrounded by aromatic amino acid residue, i.e., Phe72, Phe336, Phe732, Phe978, and Phe983. This pose had binding energy of -6.06 $\mathrm{kcal} / \mathrm{mol}$. In this study, the residues involved in binding at Hoechst 33342 and rhodamine B were not spatially well separated. There were some binding residues that contacted with rhodamine B presenting in Hoechst 33342 binding pocket. A similar observation was also reported by Kim et $a l{ }^{27}$, where they observed the top-ranked docking Hoechst 33342 poses interacted with the assigned R-site. Fluorescence resonance energy transfer (FRET) studies pointed out that the Hoechst 33342 binding site is likely located 10-14 A below the membrane surface, within the cytoplasmic leaflet of the membrane ${ }^{28}$. However, binding region regarding to the topranked pose of Hoechst 33342 was unsuccessful detected. Therefore, local docking was performed in a small area around that proposed binding sites (Figure 4C). It was observed that Trp232, Ala233, Ala295, Ser298, Ile299, Phe728, Phe983, Ala987 and Gln990 formed van der Waals contacts with Hoechst 33342, while Gln725 formed a hydrogen bond with the protonated nitrogen atom of the piperazine part of the Hoechst 33342. In addition, the benzimidazole moieties of Hoechst 33342 formed aromatic $\pi-\pi$ interactions with Phe303 and Phe343. The complex yielded a free energy of binding of $-5.35 \mathrm{kcal} / \mathrm{mol}$. The location was still closed to rhodamine binding site, but buried more deeply into the cytoplasmic membrane. Based on a pharmacophore model proposed by Pajeva et al. ${ }^{29}$, Phe303, Ile306 and Tyr307 were identified as a part of the H-site, in which the location was closed by our observations. Note that more experimental data are needed to validate the results for H-site. Furthermore, the docking results with Hoechst 33342 indicated that it oriented approximately parallel with the trans-membrane helixes, correlated well with the previous study ${ }^{28}$.

Docking analysis of colchicine demonstrated that it was located at the hydrophobic pocket of P-gp, where Ile340, Phe343, Leu339, Phe983, and Met986 contributed to the drugbinding pocket (Figure 4D). Binding energy of this interaction was $-4.57 \mathrm{kcal} / \mathrm{mol}$. Detailed analysis indicated that the 10-methoxy group formed hydrogen bond interaction with Tyr953, In addition, 2-, 3-methoxy groups formed van der Waals interactions with Phe336, Leu339 and Phe983. It was also observed that colchicine did not bind at the $\mathrm{H}$-site as reported by Shapiro and Ling ${ }^{23}$, but it located at the R-site. However, this was in accordance with several cysteine scanning mutagenesis studies ${ }^{28,30}$ indicating that Leu339, Ala342, Leu975, Va1982, and 
Ala985, which were reported as belonging to $\mathrm{R}$-site, formed a part of the colchicine binding pocket on P-gp. It was confirmed that the orientation pattern of colchicine in the predicted complexes correlated well with the experi- mental results. From above data, it was indicated that the generated human P-gp homology model was reliable and it could be used as a structural template to predict the drug binding sites on P-gp.

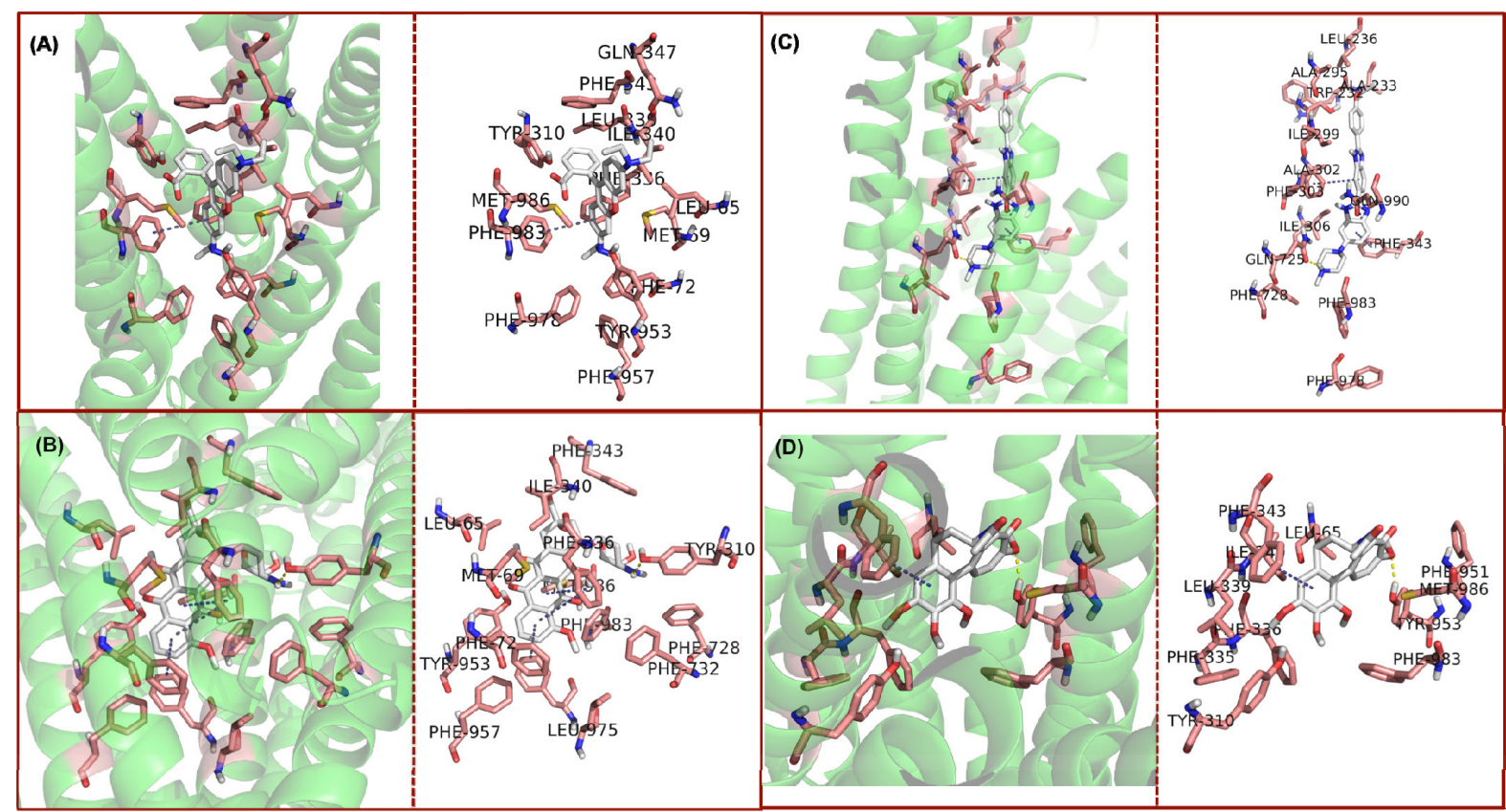

Figure 4. Binding mode of rhodamine B (A), daunorubicin (B), Hoechst 33342 (C), and colchicine (D). Detailed molecular interactions are shown in the right-hand panels. Important amino acid residues and ligands are depicted as stick model. The following color scheme is used for protein (hydrogen atoms, not involved in hydrogen bonding, are omitted for clarity), carbon in salmon pink, nitrogen in blue, oxygen in red, sulfur in yellow. The same color scheme is applied for ligands except carbon and hydrogen atoms are represented in gray and white, respectively. The hydrogen bonds are indicated by yellow dotted lines while the aromatic $\pi-\pi$ interaction is indicated by a navy blue dotted line. (For interpretation of the references to color in this figure legend, the reader is referred to web version of this article).

Additionally, verapamil was subjected to molecular docking study. Verapamil was one of P-gp inhibitors (or more correctly referred to as a P-gp modulator), which was commonly used in several experiments to localize the drug-binding pocket on P-gp ${ }^{31}$. Here, the scope was doubled: to access the homology model quality, and to determine verapamil binding site including its degree of overlapping with other drug binding sites. Verapamil was found to interact with both the $\mathrm{H}$ - and R-sites of $\mathrm{P}-\mathrm{gp}^{32}$. Cysteine-scanning mutagenesis studies by Loo et al. indicated that Leu65, Ile306, Ser222, Leu339, Ala342, Phe728, Ile868, Leu975, Tyr982, Gly984, and Ala985 formed part of the verapamil drug-binding pocket ${ }^{31,33-34}$. In silico analysis suggested that Leu65, Met69, Ile306, Tyr307, Tyr310, Phe336, Leu339, Ile340, Phe343, Gln725, Phe728, Tyr953, Phe983, and Met986 involved in the interaction of human P-gp with verapamil (Figure 5A). The results demonstrated with a good agreement with mutation experiments. Detailed analysis revealed that the methoxy groups formed van der Waals contacts with 
Leu65, Met69, Gln347, Pro350, Tyr953, and Phe983. The aromatic moieties of the ligand were surrounded by nonpolar amino acids such as Met69, Phe72, Phe336, Ile340, Phe343, Phe978, and Phe983. Best ranked docking poses for verapamil showed binding energy of $-4.16 \mathrm{kcal} /$ mol. Verapamil was observed to preferentially interact with the R-site of P-gp, similar to that of rhodamine and colchicine. This was agreed with photolabeling experiments with $\left[{ }^{3} \mathrm{H}\right]$ azidopine suggested that verapamil interacted with P-gp in the similar location as colchicine ${ }^{35}$. Furthermore, Loo et al. reported the binding region of verapamil and R-site were very similar despite the amino acid residues contributing to each site were not consistent. Therefore, it could be implied from our study and previous experiment data that verapamil binding site and R-site had some overlapping parts on P-gp. Nevertheless, verapamil was often mentioned in the literatures as modulator, it served also as substrate for $\mathrm{P}-\mathrm{gp}^{36}$. This finding might help to explain the ability of the P-gp to recognize verapamil as a substrate.

The model was then extended to characterize the binding mode of known anticancer substrate, vinblastine. Docking results revealed that Leu65, Met69, Tyr310, Ile340, Phe343, Thr945, Met949, Tyr950, Tyr953, Phe983, and Met986 involved in the interaction with P-gp, giving a binding energy of -8.61 $\mathrm{kcal} / \mathrm{mol}$. This finding qualitatively agreed with the previous studies demonstrated that arginine mutation at Gly64, Leu65, Met68, Ile868, and Thr945 residues and alanine mutation at Phe335 residue decreased the affinity of P-gp for vinblastine $^{37-38}$. From docking results, oxygen atom of the methyl-ester moiety and the hydroxyl group of the vindoline fragment formed hydrogen bonding interactions with Gln946. In addition, vindoline moiety generated a van der Waals interaction with Leu65, Ile340, Gln347, and Gln946. The hydroxyl group of the catharanthine moiety formed a hydrogen bond with the hydroxyl group of Tyr953. Furthermore, the indole ring in the catharanthine moiety formed a $\pi-\pi$ stacking interaction with Phe343 (Figure 5B). Interestingly, the docking results indicated that vinblastine appeared to lie close to verapamil drug binding pocket. The findings of our study were in agreement with mutation studies, indicating vinblastine and verapamil shared a drug binding site ${ }^{39}$. A different binding affinity between verapamil $(-4.16 \mathrm{kcal} / \mathrm{mol})$ and vinblastine $(-8.61 \mathrm{kcal} / \mathrm{mol})$ was also observed. It was, accordingly, assumed that verapamil might act as a non-competitive inhibitor for a vinblastine binding site on P-gp. This would aid in understanding how verapamil, an inhibitor of P-gp, effectively enhance cytotoxicity of vinblastine against $\mathrm{P}$-gp-mediated MDR.

\section{CONCLUSIONS}

In this study, a homology model of human P-gp based on refined crystal structure of murine P-gp (PDB entry: 4M1M) was developed. The Ramachandran plot, Z-score value, and QMEAN confirmed the reliability of the model. Substrate rhodamine B, Hoechst 33342, colchicine, and daunorubicin were subjected to blind docking experiments and their binding modes showed a good correlation with experimental data. Based on the results of this study, we concluded that the improve crystal structural model of murine P-gp (PDB entry: 4M1M) could be used to build a human P-gp model with confidence. Moreover, in silico investigation of an anticancer P-gp substrate drug vinblastine indicated that vinblastine and verapamil had an overlapping binding site within the P-gp. It could aid the experiment observations on the drug interaction between these compounds. In-depth detailed analysis of human P-gp substrates interaction and mechanism of $\mathrm{P}$-gp are in progress in our laboratory.

\section{ACKNOWLEDGMENTS}

We acknowledge support from Faculty of Pharmacy, Mahidol University and Mahidol University under the National Research Universities Initiative. 


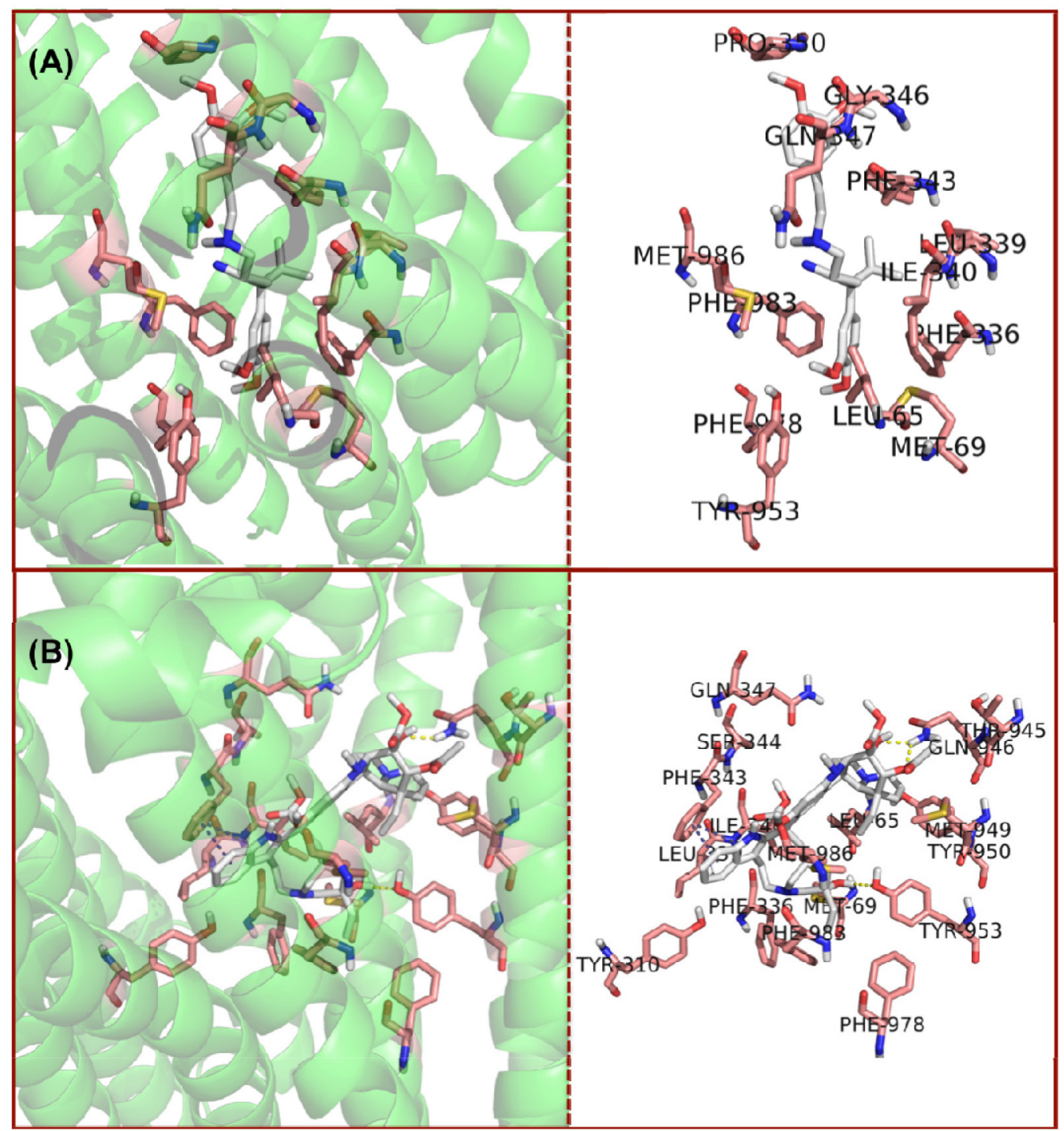

Figure 5. Model for binding of verapamil (A) and vinblastine (B) within P-gp homology model. The color scheme is the same as that in Figure 4.

\section{REFERENCES}

1. Zhou SF. Structure, function and regulation of P-glycoprotein and its clinical relevance in drug disposition. Xenobiotica. 2008;38 (7-8):802-32.

2. Wong K, Ma J, Rothnie A, Biggin PC, Kerr ID. Towards understanding promiscuity in multidrug efflux pumps. Trends Biochem Sci. 2014 ;39(1):8-16.

3. Vyas VK, Ukawala RD, Ghate M, Chintha C. Homology modeling a fast tool for drug discovery: Current perspectives. Indian J Pharm Sci. 2012; 74(1):1-17
4. Shityakov S, Förster C. In silico structurebased screening of versatile P-glycoprotein inhibitors using polynomial empirical scoring functions. Adv Appl Bioinform Chem. 2014;7:1-9.

5. Li J, Jaimes KF, Aller SG. Refined structures of mouse P-glycoprotein. Protein Sci. 2014;23(1):34-46.

6. Yamaguchi H, Kidachi Y, Kamiie K, Noshita T, Umetsu H. Homology modeling and structural analysis of human P-glycoprotein. Bioinformation. 2012;8(22):1066-74.

7. Consortium TU. The Universal Protein 
Resource (UniProt) in 2010. Nucleic Acids Research. 2010;38(suppl 1):D142-D8.

8. Altschul SF, Gish W, Miller W, Myers EW, Lipman DJ. Basic local alignment search tool. J Mol Biol. 1990;215(3):403-10.

9. Webb B, Sali A. Comparative Protein Structure Modeling Using MODELLER. Curr Protoc Protein Sci. 2016 Nov; 1;86: 2.9.1-2.9.37.

10. Laskowski RA, MacArthur MW, Moss DS, Thornton JM. PROCHECK: a program to check the stereochemical quality of protein structures. J Appl Cryst. 1993;26(2):283-91.

11. Wiederstein M, Sippl MJ. ProSA-web: interactive web service for the recognition of errors in three-dimensional structures of proteins. Nucleic Acids Research. 2007; 35(supp1 2):W407-W10.

12. Benkert P, Künzli M, Schwede T. QMEAN server for protein model quality estimation. Nucleic Acids Research. 2009;37(suppl 2): W510-W4.

13. Pollastri G, McLysaght A. Porter: a new, accurate server for protein secondary structure prediction. Bioinformatics. 2005; 21(8):1719-20.

14. Word JM, Lovell SC, Richardson JS, Richardson DC.Asparagine and glutamine: using hydrogen atom contacts in the choice of side-chain amide orientation1. J Mol Biol. 1999;285(4):1735-47.

15. O'Boyle NM, Banck M, James CA, Morley C, Vandermeersch T, Hutchison GR. Open Babel: An open chemical toolbox. J Cheminform. 2011;3(1):33.

16. Morris GM, Huey R, Lindstrom W, Sanner MF, Belew RK, Goodsell DS, et al. AutoDock4 and AutoDockTools4: Automated docking with selective receptor flexibility. J Comp Chem. 2009;30(16):2785-91.

17. Gasteiger J, Marsili M. Iterative partial equalization oforbitalelectronegativity - a rapid access to atomic charges. Tetrahedron. 1980;36(22):3219-28.

18. Hetényi C, van der Spoel D. Efficient docking of peptides to proteins without prior knowledge of the binding site. Protein Sci. 2002;11(7):1729-37.
19. Delano WL. The PyMOL Molecular Graphics System 2002. Available from: http://www.pymol.org.

20. Benkert P, Tosatto SCE, Schomburg D. QMEAN: A comprehensive scoring function for model quality assessment. Proteins. 2008;71(1):261-77.

21. Sim H-M, Bhatnagar J, Chufan EE, Kapoor K, Ambudkar SV. Conserved walker A cysteines 431 and 1074 in human P-glycoprotein are accessible to thiol-specific agents in the apo and ADP-vanadate trapped conformations. Biochemistry. 2013;52(41): 7327-38.

22. Loo TW, Bartlett MC, Clarke DM. Processing mutations disrupt interactions between the nucleotide binding and transmembrane domains of P-glycoprotein and the cystic fibrosis transmembrane conductance regulator (CFTR). J Biol Chem. 2008;283(42):28190-7.

23. Shapiro AB, Ling V. Positively cooperative sites for drug transport by P-Glycoprotein with distinct drug specificities. Eur J Biochem. 1997;250(1):130-7.

24. Loo TW, Clarke DM. Location of the rhodamine-binding site in the human multidrug resistance P-glycoprotein. J Biol Chem. 2002;277(46):44332-8.

25. Loo TW, Bartlett MC, Clarke DM. Suppressor mutations in the transmembrane segments of P-glycoprotein promote maturation of processing mutants and disrupt a subset of drug-binding Sites. J Biol Chem. 2007;282 (44):32043-52.

26. Sharom FJ, Lugo MR, Eckford PDW. New insights into the drug binding, transport and lipidflippase activities of the P-Glycoprotein multidrug transporter. J Bioenerg Biomembr. 2005;37(6):481-7.

27. Kim N, Shin J-M, No KT. In silico study on the interaction between P-glycoprotein and its inhibitors at the drug binding. Bull Korean Chem Soc. 2014;35(8):2317-25.

28. Qu Q, Sharom FJ. Proximity of bound Hoechst 33342 to the ATPase catalytic sites places the drug binding site of P-glycoprotein within the cytoplasmic membrane leaflet. Biochemistry. 2002;41 (14):4744-52. 
29. Pajeva IK, Globisch C, Wiese M. Structurefunction relationships of multidrug resistance P-Glycoprotein. J Med Chem. 2004;47(10): 2523-33.

30. Loo TW, Clarke DM. Identification of residues within the drug-binding domain of the human multidrug resistance $\mathrm{P}$-glycoprotein by cysteine-scanning mutagenesis and reaction with dibromobimane. J Biol Chem. 2000;275(50):39272-8.

31. Loo TW, Clarke DM. Mutational analysis of $\mathrm{ABC}$ proteins. Arch Biochem Biophys. 2008;476(1):51-64.

32. Al-Shawi MK, Polar MK, Omote H, Figler RA. Transition state analysis of the coupling of drug transport to ATP hydrolysis by P-glycoprotein. J Biol Chem. 2003;278 (52):52629-40.

33. Loo TW, Clarke DM. Defining the drugbinding site in the human multidrug resistance P-glycoprotein using a methanethiosulfonate analog of verapamil, MTS-verapamil. J Biol Chem. 2001;276(18):14972-9.

34. Loo TW, Bartlett MC, Clarke DM. Permanent activation of the human P-glycoprotein by covalent modification of a residue in the drug-binding site. J Biol Chem. 2003;278
(23):20449-52.

35. Safa AR, Glover CJ, Sewell JL, Meyers MB, Biedler JL, Felsted RL. Identification of the multidrug resistance-related membrane glycoprotein as an acceptor for calcium channel blockers. J Biol Chem. 1987;262 (16):7884-8.

36. Kim RB. Drugs as P-glycoprotein substrates, inhibitors, and inducers. Drug Metab Rev. 2002;34(1-2):47-54.

37. Loo TW, Bartlett MC, Clarke DM. Identification of residues in the drug translocation pathway of the human multidrug resistance P-glycoprotein by arginine mutagenesis. J Biol Chem. 2009;284(36):24074-87.

38. Loo TW, Bartlett MC, Clarke DM. Arginines in the first transmembrane segment promote maturation of a P-glycoprotein processing mutant by hydrogen bond interactions with tyrosines in transmembrane Segment 11. J Biol Chem. 2008;283(36):24860-70.

39. Loo TW, Clarke DM. Identification of residues in the drug-binding domain of human P-glycoprotein. Analysis of transmembrane segment 11 by cysteine-scanning mutagenesis and inhibition by dibromobimane. J Biol Chem. 1999;274(50):35388-92. 